\title{
A escola prevenindo a obesidade infantil através de políticas de saúde alimentar: uma revisão narrativa
}

\author{
The school preventing childhood obesity through food health policies: a narrative review
}

La escuela que previene la obesidad infantil a través de políticas de salud alimentaria: una revisión narrativa

Mônica Cristina de Orequio ${ }^{1 *}$, Alessandra Ribeiro de Oliveira ${ }^{1}$, Marcus Vinicius Pena Abranches Pacheco ${ }^{1}$, Daniel Franz Reich Magalhães ${ }^{1}$, Marcella de Oréquio Fernandes Machado', Aline Carvalho Moreira da Silva ${ }^{1}$, Evan Pereira Barreto ${ }^{1}$, Cláudia Mariano Simões ${ }^{1}$, Angérica Maurício de Souza Gomes ${ }^{1}$, Valéria Cristina Calvi Fontana².

\section{RESUMO}

Objetivo: Através da busca bibliográfica sobre a obesidade infantil através de políticas escolares para uma alimentação saudável e de saúde. Revisão bibliográfica: É notório que a obesidade infantil tem se tornado um problema cada vez mais grave no Brasil, já que após os anos 80 o acesso a alimentos pouco saudáveis aumentou com o fortalecimento do poder de compra do brasileiro. Com isso, a disparada de doenças que são causadas ou agravadas pela obesidade, tiveram uma alta sem precedentes e geraram preocupações aos profissionais da nutrição entre outros profissionais da saúde. A busca por soluções bate as portas das escolas, local destinado a promover o conhecimento científico e básico, e que pode se tornar uma forte aliada da luta para prevenir doenças futuras através de instruções sobre alimentações saudáveis e equilibradas. Considerações finais: Compreende-se, portanto, que a promoção de saúde alimentar nas escolas através da instrução, é a melhor saída para garantir resultados positivos através da junção de saúde e educação no combate a obesidade infantil e as doenças causadas por ela.

Palavras-chave: Promoção da saúde dos alunos, Saúde na escola, Educação nutricional.

\section{ABSTRACT}

Objective: Through the bibliographic search on childhood obesity through school policies for healthy eating and health. Bibliographic review: It is well known that childhood obesity has become an increasingly serious problem in Brazil, since after the 1980s, access to unhealthy foods increased with the strengthening of Brazilian purchasing power. With this, the skyrocketing of diseases that are caused or aggravated by obesity, had an unprecedented rise and raised concerns for nutrition professionals among other health professionals. The search for solutions knocks the doors of schools, a place designed to promote scientific and basic knowledge, and which can become a strong ally in the fight to prevent future diseases through instructions on healthy and balanced food. Final considerations: It is understood, therefore, that the promotion of food health in schools through education, is the best way to guarantee positive results by combining health and education in combating childhood obesity and the diseases caused by it.

Keywords: Student health promotion, School health, Nutrition education.

\section{RESUMEN}

Objetivo: Mediante la búsqueda bibliográfica sobre obesidad infantil a través de políticas escolares de alimentación saludable y salud. Revisión bibliográfica: Es bien sabido que la obesidad infantil se ha convertido en un problema cada vez más grave en Brasil, ya que después de la década de 1980, el acceso a alimentos no saludables aumentó con el fortalecimiento del poder adquisitivo brasileño. Con esto, el aumento vertiginoso de las enfermedades provocadas o agravadas por la obesidad, tuvo un aumento sin precedentes y generó preocupación para los profesionales de la nutrición entre otros profesionales de la salud. La búsqueda de soluciones golpea las puertas de las escuelas, un lugar diseñado para promover el conocimiento

${ }^{1}$ Faculdade Vale do Cricaré (FVC), São Mateus - ES. *E-mail: monicacarocris@yahoo.com.br

2 Universidad Columbia del Paraguay, Assunção - PAR.

SUBMETIDO EM: $8 / 2020$

ACEITO EM: 9/2020

PUBLICADO EM: 12/2020 
científico y básico, y que puede convertirse en un fuerte aliado en la lucha para prevenir futuras enfermedades a través de instrucciones sobre alimentación sana y equilibrada. Consideraciones finales: Se entiende, por tanto, que la promoción de la salud alimentaria en las escuelas a través de la educación es la mejor forma de garantizar resultados positivos al combinar la salud y la educación en el combate a la obesidad infantil y las enfermedades que ésta provoca.

Palabras clave: Promoción de la salud estudiantil, Salud escolar, Educación nutricional.

\section{INTRODUÇÃO}

A Organização Mundial da Saúde relatou que a obesidade infantil nos países europeus aumentou cerca de $40 \%$ na última década. No Brasil, os últimos dados fornecidos pelo Instituto Brasileiro de Geografia e Estatística (IBGE) são de 2008/2009 (IBGE, 2010). Na região Sudeste os dados são mais expressivos, a taxa de excesso de peso de crianças de 5 a 9 anos é de $38,8 \%$, enquanto a taxa de excesso de peso de crianças de 10 a 19 anos é de 22,8\% (FRONTZECK LGM, et al., 2017).

Nas últimas décadas, mudanças estruturais nos hábitos alimentares de crianças e adolescentes resultaram em aumento da ingestão de gorduras e açúcares e consumo reduzido de alimentos ricos em fibras; essas mudanças nos hábitos alimentares acarretaram o aumento da prevalência de obesidade nos países em desenvolvimento.

A industrialização de alimentos foi identificada como uma das principais razões que levaram a redução da dieta saudável na maioria das pessoas no Ocidente. Outras mudanças no estilo de vida reduziram o escopo da atividade física, que está relacionada ao desenvolvimento de tecnologia e à popularização de jogos e equipamentos de vídeo, resultando em uma diminuição significativa da atividade física, que por sua vez leva à obesidade e doenças relacionadas, inclusive em crianças (OLIVEIRA CB, et al., 2012).

A depressão é considerada outra característica relacionada à dinâmica da personalidade de indivíduos obesos. A depressão infantil também pode ser um sintoma de lesões orgânicas e se desenvolve na obesidade. Isso se manifesta por apetite constante, tristeza, irritabilidade, combatividade, fadiga crônica, etc. É a principal queixa de fadiga, dependendo da intensidade e frequência, podendo apresentar sintomas de depressão e interferir na vida das crianças, resultando em distúrbios comportamentais em suas relações sociais. Crianças e adolescentes obesos têm um apetite maior, são propensos à depressão, têm relacionamentos interpessoais mais pobres, desequilíbrios emocionais aumentados e uma fraca tolerância à frustração (MENDES JOH, et al., 2019).

A obesidade pode começar em qualquer idade por razões que incluem o desmame precoce, má distribuição de alimentos durante a infância, substituição excessiva da amamentação por carboidratos e um estilo de vida sedentário. Nesse sentido, estudos realizados em cidades brasileiras demonstraram que a prevalência de sobrepeso e obesidade em crianças aumentou e demonstraram estar relacionados a certos fatores de risco, como hipertensão arterial, hipercolesterolemia e resistência à insulina. No entanto, poucos estudos acreditam que o status socioeconômico é um fator que leva ao desenvolvimento de sobrepeso ou obesidade (MIRANDA JMQ, et al., 2015).

Com o início da vida escolar, as crianças terão os hábitos alimentares afetados, que é um estágio típico de formação de hábitos e comportamentos que podem continuar na idade adulta. Desse ponto de vista, foram desenvolvidas estratégias para utilizar o potencial da escola como espaço de promoção da saúde, formando hábitos alimentares saudáveis.

Pesquisas sobre o consumo de alimentos no ambiente escolar mostram que, sejam esses alimentos comprados no refeitório da escola ou levados de casa para consumo escolar e até fornecidos por programas públicos de alimentação, os alunos ainda consomem grandes quantidades de alimentos com baixo valor nutricional e alta densidade de energia.

Além disso, há evidências de que há uma correlação positiva entre obesidade e certas características do ambiente escolar, como o fornecimento de doces e frituras e o fornecimento de máquinas ou estabelecimentos de alimentos e bebidas (ROSSI CE, et al., 2019). 
O excesso de peso é enxergado como um sério que afeta a população e pode causar doenças cardiovasculares, pressão alta, doenças respiratórias, diabetes, dislipidemia e certos tipos de tumores e outras doenças. Quanto mais intenso e precoce parecer, maior o risco de resistência e mais graves as comorbidades associadas. Vale ressaltar que, devido à discriminação infantil e à aceitação social desse problema, o excesso de peso na infância e adolescência pode causar algumas complicações psicossociais, pois muitas vezes são isoladas da sociedade e se retiram das atividades. Além dessas sérias consequências, o excesso de peso é considerado a doença que mais cresce no mundo (SOUZA MCC, et al., 2014).

Esta revisão objetiva, através da literatura existente, apresentar a realidade alimentar dos alunos brasileiros e, através de políticas públicas de saúde alimentar, propor alterações e almejar uma nova realidade para crianças e adolescentes. Também se objetiva neste estudo, chamar a atenção para possíveis soluções que podem ser desenvolvidas na escola, através de instrução alimentar, aproximação do aluno com alimentos nutricionalmente ricos e o despertar da importância de se prevenir doenças na infância.

\section{REVISÃO BIBLIOGRÁFICA}

Como se sabe, a prevalência de obesidade está aumentando e é considerada uma epidemia mundial e um problema global de saúde pública. O Portal da Saúde relata que metade da população brasileira está acima do peso e, segundo o IBGE (2010), os números repetem os dados do estado do Rio Grande do Sul. Uma pesquisa com 1.666 escolares de 7 a 17 anos de Santa Cruz do Sul mostrou que 26,7\% das crianças avaliadas estavam com sobrepeso ou obesidade, e aquelas com um teor de gordura superior a moderadamente alto eram 35,9\%. Nesse sentido, é necessário refletir sobre alternativas sobre os considerados fatores de risco para o desenvolvimento da obesidade infantil e, ao mesmo tempo, solucionar o problema da falta de políticas públicas efetivas para prevenir e tratar a doença (DURÉ ML, et al., 2015).

Os propulsores etiológicos da obesidade são combinados com fatores biológicos e ambientais, incluindo hábitos alimentares, predisposição genética, estilo de vida, status socioeconômico e fatores psicológicos. Entre os fatores relacionados aos hábitos alimentares, o mais importante é ingerir alimentos com baixo valor nutricional e alta densidade energética.

Como parte da política nacional de promoção da saúde, o Ministério da Saúde brasileiro desenvolveu uma série de estratégias para promover uma alimentação saudável nas escolas, para incentivar a comunidade escolar a observar hábitos alimentares saudáveis. No entanto, poucos estudos avaliaram os lanches consumidos por crianças em idade escolar com base em diretrizes de programas governamentais e até poucos estudos nacionais determinaram a diferença entre os lanches trazidos em casa e os comprados na cantina da escola e seu impacto no desenvolvimento de sobrepeso ou lanches influências (ROSSI CE, et al., 2019).

Além de prever a obesidade na idade adulta, o excesso de peso e a obesidade infantil também podem prever complicações. Essa situação preocupa o país porque aumenta os custos socioeconômicos e cria sérios problemas de saúde pública. Com o forte apoio do governo brasileiro, a Assembleia Mundial da Saúde aprovou a Estratégia Global de Alimentação, Exercício Físico e Saúde em 2004. A estratégia afirma que, para resolver esse problema, são necessárias políticas públicas que incentivem a alimentação saudável e o exercício físico. Exemplos dessa estratégia são: medidas financeiras para tornar mais acessíveis alimentos saudáveis, como frutas e legumes, restrições à publicidade de alimentos nocivos e planejamento urbano para exercícios físicos diários (VERTELO MM, 2011).

O percentual de massa gorda (PMG) também é um método de avaliação clínica da obesidade, que corresponde à gordura total expressa como uma porcentagem do peso corporal total. Este parâmetro pode identificar objetivamente indivíduos obesos com maior risco metabólico. Ainda estão em andamento pesquisas sobre a determinação correta da PMG das crianças e a definição precisa dos valores de obesidade em diferentes idades. A obesidade está associada a uma variedade de doenças crônicas que diminuem a vida útil, como doenças cardiovasculares, diabetes e doenças ósseas e articulares. Essas doenças costumavam ser características dos adultos e agora são mais comuns em crianças (VENÂNCIO P, et al., 2012). 
Medidas devem ser tomadas para prevenir a obesidade infantil. Essas medidas já podem ser tomadas na vida no útero, incluindo a promoção da saúde das gestantes, a identificação de fatores de risco para a saúde de gestantes e crianças e a orientação de hábitos de vida conducentes a um peso saudável. Nos primeiros anos de vida, é importante fornecer orientações alimentares apropriadas e específicas para que os cuidadores não apenas entendam quais alimentos devem ser consumidos, mas também como preparar os alimentos, e a quantidade e a idade desses alimentos. Além disso, é importante que famílias, escolas e comunidades inteiras participem de programas coletivos para melhorar a saúde das crianças (CARVALHO EAA, et al., 2013).

Em relação ao envolvimento dos pais no tratamento de pacientes pediátricos com sobrepeso e obesidade, há sinais de que os hábitos de vida familiar são extremamente importantes na perda de peso, assim como é imprescindível analisar a relação de todo o ambiente social de convivência da criança com os distúrbios alimentares.

A escola, que se identifica como ambiente de promoção do conhecimento e local socializador, pode se tornar um grande pesadelo, já que as práticas de respeito e aceitação das diferenças, que não são difundidas nos lares brasileiros, acabam sendo completamente desprezadas nos momentos de inteiração. A realização necessária para se alcançar resultados satisfatórios e o desenvolvimento de políticas educacionais alimentares, é a instrução alimentar através de disciplinas específicas como ciências e educação física, além da promoção de bons hábitos no cotidiano do aluno (LINHARES FMM, et al., 2016).

\section{Problemas que desencadeiam a obesidade infantil}

A obesidade é uma doença complexa, com graves impactos sociais e psicológicos, afetando quase todas as idades e grupos socioeconômicos. É considerada uma doença não infecciosa com as seguintes características: longo período de incubação, longo curso assintomático, o curso clínico geralmente é lento, manifestações clínicas permanentes e de longo prazo, incluindo período de remissão e exacerbação e uma variedade de medições, além de uma forte Composição ambiental. Vale ressaltar que, em muitos casos, não há dor física, mas há dor e dor psicossocial.

A obesidade é uma doença crônica em desenvolvimento, que apresenta um certo grau de armazenamento de gordura e riscos à saúde devido à sua semelhança com várias complicações metabólicas e fatores de risco genéticos e ambientais. Segundo a Organização Mundial da Saúde (OMS), existem mais de um bilhão de adultos com excesso de peso, pelo menos 300 milhões deles são obesos (LOPES PCS, et al., 2010).

$\mathrm{Na}$ literatura, acredita-se que a causa da obesidade é muito complexa e apresenta características multifatoriais. Portanto, envolve uma série de fatores, incluindo fatores históricos, ecológicos, políticos, socioeconômicos, sociopsicológicos, biológicos e culturais. No entanto, deve-se ressaltar que, de maneira geral, os fatores mais estudados para a obesidade são fatores biológicos relacionados ao estilo de vida, principalmente em termos de dieta binomial e atividade física. A pesquisa aborda questões relacionadas a maior suprimento de energia na dieta e menor atividade física devido à falta de atividade física, que constitui um estilo de vida comumente conhecido por estilo ocidental contemporâneo (WANDERLEY EN e FERREIRA VA, 2010).

Observando os estudos mais profundos depara-se como que a genômica, o genoma completo de um organismo é estudado, e a transcriptômica e a proteômica que constituem a "plataforma" também são usadas para estudar o RNA e a proteína do organismo, respectivamente. Entre elas, destacam-se as novas áreas de pesquisa no campo da ciência nutricional: a genômica nutricional, que inclui a genética nutricional para estudar a variação genética (genótipos) e sua resposta aos padrões alimentares no contexto da saúde/doença e para estudar a expressão nutricional dos genes (genética).

Ainda no campo da genética, temos epigenômica nutricional que pode estudar alterações epigenéticas que afetam a expressão gênica, mas não são devidas a alterações na sequência base do DNA e sim alterações na atividade pós-transcricional de genes como metilação e acetilação (PEREIRA V, et al., 2019).

Existem várias maneiras de classificar e diagnosticar a obesidade. Atualmente, um dos métodos mais usados atualmente é baseado na gravidade do excesso de peso, calculada pelo índice de massa corporal (IMC ou índice de Quetelet). O impacto genético na obesidade pode ser causado pela herança de um único 
gene. A herança de um único gene é determinada por um ou vários genes e sua expressão não é afetada pelo ambiente ou dificilmente afetada pelo ambiente. Pode ser autossômica ou sexualmente relacionada, mas dominante ou recessiva. Os fatores genéticos desempenham um papel importante na manutenção do peso corporal, porque existem genes envolvidos na regulação do gasto energético, apetite, metabolismo lipídico, produção de calor, diferenciação celular e efeitos sinérgicos (PAZ CP, et al., 2017).

A relevância da depressão no contexto clínico é bem conhecida, o que impõe grandes restrições a indivíduos, famílias e sociedade. Observa-se na literatura as diferenças entre os estudos enquanto estudavam a matéria disponível e descobriram que alguns estudos apontaram que a obesidade aumenta o risco de desenvolver sintomas depressivos, enquanto outros indicam que a obesidade reduz o risco de sintomas depressivos. Entretanto, outros relataram que a obesidade não afeta o risco de depressão. Acerca da influência da obesidade no cotidiano da pessoa, há que se considerar as implicações psicossociais e as barreiras impostas na convivência, já que os estigmas da sociedade acabam afugentando e reprimindo pessoas obesas (ROCHA C e COSTA E, 2012).

O risco de obesidade é influenciado pela genética e existem vários potencializadores relacionados à obesidade. No entanto, a prevalência da obesidade aumentou rapidamente nas últimas décadas, não podendo ser atribuída apenas à expressão gênica, mas pode ser explicada pela interação entre a suscetibilidade genética e o ambiente causador da obesidade. O surgimento da obesidade, além de casos raros de indivíduos com síndrome genética, deve-se às consequências de longo prazo dos desequilíbrios no balanço energético determinados por fatores ambientais (como ingestão de alimentos e atividade física), bem como por fatores biológicos. Efeitos causados também por certos hormônios e neurotransmissores (leptina, grelina e adiponectina) que afetam o apetite, à saciedade e a distribuição de gordura no corpo (GARCIA AR, 2013).

Considerando a dificuldade e a alta taxa de falhas do tratamento da obesidade em crianças e adolescentes, é muito importante determinar uma estratégia eficaz de prevenção da obesidade. $O$ mais simples é o medicamento que não fornece efeitos colaterais. Nesse sentido, o aleitamento materno é uma estratégia possível para prevenir a obesidade infantil, que é uma medida protetora aprovada pela Organização Mundial da Saúde. Muitas hipóteses foram propostas para explicar por que a amamentação pode proteger as crianças da obesidade. O mecanismo de proteção envolve todos os aspectos, da composição específica e única do leite humano à influência de fatores ambientais e comportamentais, como nível socioeconômico, educação materna, dieta e exercício físico (SIQUEIRA RS e MONTEIRO CA, 2007).

\section{Propostas para solucionar a obesidade infantil através da escola}

Estudos indicam a prevalência de sobrepeso e obesidade em crianças e adolescentes. Vale ressaltar o fato de que, em certas etapas da vida, menos de $10 \%$ dos casos de sobrepeso e obesidade são causados por fatores endócrinos, e estudos longitudinais demonstraram que a obesidade infantil costuma prevalecer na idade adulta. Na perspectiva das estratégias educacionais, o objetivo é minimizar o impacto negativo dos estilos de vida que afetam a saúde e a qualidade de vida dos jovens em espaços e instituições sociais, sendo as escolas um local privilegiado para solucionar esse problema (PAIXÃO JA, et al., 2016).

Mais especificamente, a educação física é um campo do conhecimento que utiliza o movimento humano como eixo norteador de vários comportamentos físicos, e é possível aumentar a conscientização dos alunos sobre a importância de adotar um estilo de vida ativo. Trata-se de uma ação educativa valiosa, na qual é aberto espaço para lidar com riscos como a obesidade, situação que pode se tornar o oposto de obter e manter a saúde e a qualidade de vida. Essas ações educacionais englobam inúmeros campos do conhecimento que não estão apenas localizados na disciplina de educação física, mas também nas disciplinas da área de ciências, entre inúmeras outras correlações que se pode fazer com o cotidiano escolar, como a hora da merenda e os horários reservados aos lanches (PAIXÃO JA, et al., 2016).

As diretrizes para promover a alimentação saudável nas escolas foram recentemente desenvolvidas como elementos a serem considerados nos programas políticos e de ensino das unidades escolares. A construção do projeto implica a participação de profissionais da educação, escolas e comunidades locais, e visa esclarecer a gestão democrática entre escolas e comunidades. Nesse sentido, o Ministério da Saúde e o 
Ministério da Educação são conjuntamente responsáveis por garantir a implementação da Política Nacional de Alimentação e Nutrição e, portanto, a implementação da Política Nacional de Promoção da Saúde nas escolas, de acordo com os padrões de implementação do Programa Nacional de Alimentação Escolar (PNAE). Este último visa atender as necessidades nutricionais dos alunos durante a escola e promover hábitos alimentares saudáveis nesse contexto (CERVATO-MANCUSO AM, et al., 2013).

Nessa abordagem, o PNAE se torna uma possibilidade de redefinir as ações formuladas pela escola e pode desempenhar um papel estratégico na mudança de hábitos alimentares da escola. Também pode promover a educação e nutrição em saúde e embasar a prática da educação em nutrição a partir de uma perspectiva mais ampla da cidadania. Dessa forma, as diferentes experiências da escola no Brasil foram consolidadas de acordo com a proposta de iniciativa para a promoção da saúde na escola e incentivo ao esporte.

A proposta é aprovada pela Organização Pan-Americana da Saúde (OPAS) para orientar a prática do desenvolvimento - relacionamento interpessoal construtivo e harmonioso. A relação de comportamentos humanos saudáveis promove atitudes em relação à saúde e atitudes positivas também colabora para o desenvolvimento de ações que permitam expandir significativamente o papel da merenda escolar na promoção da educação em saúde e nutrição (CUNHA E, 2010).

As escolas desempenham um papel importante no treinamento cívico, incentivando o autogoverno, exercendo direitos e obrigações, controlando o estado de saúde e a qualidade de vida e adquirindo comportamentos e atitudes, para que os espaços que promovem a saúde sejam considerados saudáveis. As atividades educacionais que promovem a saúde escolar e, em particular, a promoção da alimentação saudável (PAS), representam possibilidades específicas na comunidade escolar que têm impacto no desenvolvimento da saúde, autoestima, comportamento e habilidades para a vida de todos os membros. Tais atividades devem ser implementadas por meio de ações intersetoriais e horizontais, e o tema deve ser incorporado ao plano de ensino da escola (CAMOZZI ABQ, et al., 2015).

Propor modificações no ambiente educacional a fim de combater a obesidade infantil e aumenta a vida útil dos alunos, é um consenso entre profissionais da nutrição e educadores das áreas específicas. Entretanto, cuidar da saúde alimentar e prevenir o sedentarismo, além do sobrepeso, não significa estabelecer padrões de beleza e estética com base no pensamento social atrasado. Não se trata de políticas estéticas, e sim de cuidados com a saúde e prevenção de futuras morbidades em decorrência da má alimentação e do excesso de peso. A questão estética não deve ser tratada, já que o problema está concentrado na visão social que é inclinada aos rótulos e acusadora de diferenças. A instrução nesses casos é um debate profundo sobre diferenças e respeito mútuo, instruindo cuidados e inspirando reprovação ao bullying (NASCIMENTO VG, et al., 2011).

\section{CONSIDERAÇÕES FINAIS}

Os problemas relacionados a obesidade infantil estão diretamente ligados a questão genética, mas também a questões sociais e a ausência de instrução e conhecimento. A utilização do ambiente escolar é indispensável para solucionar o problema aqui apresentado, já que a escola funciona como casa de conhecimento e exploração científica, e pode ampliar a margem de conhecimento sobre as questões que levam as crianças a se alimentar de maneira errada. As políticas públicas de combate a obesidade infantil devem começar desde a gestação e avançar até o período limite da infância, já que estudos apontam que a obesidade infantil na grande parte dos casos é seguida de obesidade na adolescência e na vida adulta.

\section{REFERÊNCIAS}

1. CAMOZZI $A B Q$, et al. Promoção da Alimentação Saudável na Escola: realidade ou utopia?. Cad. Saúde Coletiva, $2015 ; 23(1): 32-37$.

2. CARVALHO EAA, et al. Obesidade: aspectos epidemiológicos e prevenção. Rev Med Minas Gerais, 2013; 23(1): 7482.

3. CERVATO-MANCUSO AM, et al. O papel da alimentação escolar na formação dos hábitos alimentares. Revista Paulista de Pediatria, 2013; 31(3): 324-330. 
4. CUNHA E. A alimentação orgânica e as ações educativas na escola:diagnóstico para a educação em saúde e nutrição. Ciência \& Saúde Coletiva, 2010; 15(1): 39-49.

5. DURÉ ML, et al. A obesidade infantil: um olhar sobre o contexto familiar, escolar e da mídia. R. Epidemiol. Control. Infec., Santa Cruz do Sul, 2015; 5(4): 191-196.

6. FRONTZECK LGM, et al. Obesidade infantil: compreender para melhor intervir. Revista da Abordagem Gestáltica, 2017; 23(2): 167-174.

7. GARCIA AR. Influência do Aleitamento Materno na Prevenção da Obesidade em Idade Pediátrica. Revista Nutrícias, 2013; 16(1): 26-29.

8. IBGE. 2010. INSTITUTO Brasileiro de Geografia e Estatística. Portal IBGE. Disponível em: https://www.ibge.gov.br/. Acesso em: 8 de jul. 2020.

9. LINHARES FMM, et al. Obesidade infantil: influência dos pais sobre a alimentação e estilo de vida dos filhos. Temas em Saúde, 2016; 16(2): 460-481.

10. LOPES PCS, et al. Fatores de risco associados à obesidade e sobrepeso em crianças em idade escolar. Revista Brasileira de Enfermagem, 2010; 63(1): 73-78.

11. MENDES JOH, et al. Características psicológicas e relações familiares na obesidade infantil: uma revisão sistemática. Revista da SBPH, 2019; 22(2): 228-247.

12. MIRANDA JMQ, et al. Prevalência do sobrepeso e obesidade infantil em instituições de ensino: públicas vs. Privadas. Rev Bras Med Esporte, 2015; 21(2): 104-107.

13. NASCIMENTO VG, et al. Risco de sobrepeso e excesso de peso em crianças de pré-escolas privadas e filantrópicas. Revista da Associação Médica Brasileira, 2011; 57(6): 657-661.

14. OLIVEIRA CB, et al. Obesidade infantil, prevenção e intervenção. Uma revisão de literatura. EFDeportes.com, 2012; 17(167): 1-9.

15. PAZ CP, et al. Obesidade: considerações sobre os fatores genéticos. Rev. Interd. Ciên.Saúde, 2017; 4(2): $106-112$.

16. PEREIRA V, et al. Fatores genéticos, epigenómicos, metagenómicos e cronobiológicos da obesidade. Acta portuguesa de nutrição, 2019; 17(1): 22-28.

17. ROCHA C, COSTA E. Aspectos psicológicos na obesidade mórbida: Avaliação dos níveis de ansiedade, depressão e do auto-conceito em obesos que vão ser submetidos à cirurgia bariátrica. Análise Psicológica, 2012; 30(4): 451 -466.

18. ROSSI CE, et al. Fatores associados ao consumo alimentar na escola e ao sobrepeso/obesidade de escolares de 7 10 anos de Santa Catarina, Brasil. Ciência \& Saúde Coletiva, 2019; 24(2): 443-454.

19. SIQUEIRA RS, MONTEIRO CA. Amamentação na infância e obesidade na idade escolar em famílias de alto nível socioeconômico. Rev Saúde Pública, 2007; 41(1): 5-12.

20. SOUZA MCC, et al. Fatores associados à obesidade e sobrepeso em escolares. Texto Contexto Enferm, 2014; 23(3): 712-719.

21. VENÂNCIO P, et al. Obesidade infantil... um problema cada vez mais actual. Revista Portuguesa de Medicina Geral e Familiar, 2012; 28(6): 410-416.

22. VERTELO MM. Estudos e Estratégias para prevenção e tratamento da obesidade infantil no âmbito escolar. Monografia (Curso de Licenciatura em Biologia). Universidade de Brasília/Universidade Estadual de Goiás, Brasília, $2011 ; 30 \mathrm{p}$.

23. WANDERLEY EM, FERREIRA VA. Obesidade: uma perspectiva plural. Ciência \& Saúde Coletiva, 2010; $15(1)$ : 185194. 